\title{
SAÚDE DE ATIVOS E MANUTENÇÃO POR CONDIÇÃO NA INSTRUMENTAÇÃO DE USINAS DE TRATAMENTO DE MINÉRIO DE FERRO*
}

\author{
Juliene Beatriz de Oliveira Ragonesi ${ }^{1}$ \\ Luis Gustavo Nunes Ragonesi \\ Paulo Júnior de Carvalho ${ }^{3}$ \\ Siderlei Carvalho Ribeiro Santos ${ }^{4}$ \\ Vicente Magela Reis ${ }^{5}$ \\ Vicentino José Pinheiro Rodrigues ${ }^{6}$
}

\section{Resumo}

O objetivo do trabalho é criar um método capaz de gerar indicadores de inspeção preditiva dos ativos de instrumentação, utilizando dados colhidos de recursos existentes (PIMS - Plant Information Management System) através de técnicas estatísticas. Esses indicadores serão capazes de destacar os ativos prioritários e informar para as áreas os instrumentos que necessitam de intervenções, evitando parada de ativos críticos em corretivas. O conceito de ativo crítico para o projeto é de um instrumento cuja falha paralisa a produção.

Palavras-chave: Saúde de ativos; Manutenção por condição; Indicadores de inspeção.

\section{INSTRUMENT MAINTENANCE UNDER CONDITIONS IN IRON ORE PLANTS}

\begin{abstract}
The objective is to create a method capable of generating predictive inspection indicators of instrumentation assets, using data collected from existing resources (PIMS - Plant Information Management System) through statistical techniques. Then, system will be able to highlight the major assets to be maintained and report to the areas those that need intervention, avoiding stops of critical assets in corrective. The concept of critical asset for the project is an instrument whose fail paralyzes production.
\end{abstract}

Keywords: Health assets; Maintenance under condition; Inspection indicators..

Supervisora de Automação na Vale SA, Complexo de Mariana, MG, Brasil.

Gerente de manutenção elétrica na Vale SA, Complexo de Mariana, MG, Brasil.

Supervisor de manutenção elétrica de usina na Vale SA, Complexo de Mariana, MG, Brasil.

Engenheiro de automação na Vale SA, Complexo de Mariana, MG, Brasil.

Analista de automação na Vale SA, Complexo de Mariana, MG, Brasil.

Gerente de automação na Vale SA, Complexo de Mariana, MG, Brasil. 


\section{INTRODUÇÃO}

As usinas de beneficiamento de minério de ferro da Diretoria de Ferrosos Sudeste [1] da Vale em Minas Gerais possuem histórico de grande dependência de instrumentação e automação para controle de seus processos e gerenciamento de informações de produção.

A tecnologia dos controladores lógicos programáveis foi adotada pela indústria da mineração em nosso estado a partir da década de 80 e este evento criou um mercado florescente de empresas de engenharia, integradoras de sistemas e fabricantes de equipamentos voltados para a mineração, fazendo da cidade de Belo Horizonte um polo importante da indústria da automação no Brasil.

O uso da automação evoluiu no que diz respeito à configuração de equipamentos e arquitetura de redes, armazenamento de dados para exibição de tendências, sintonia de malhas de controle, integração com sistemas (PIMS \& MES), sistemas supervisórios mais amigáveis e com mais recursos, sempre beneficiando a área de operação.

O trabalho de saúde de ativos nasceu em decorrência de uma necessidade da manutenção de elétrica e instrumentação proporcionar a maior confiabilidade possível aos ativos de automação que monitoram os equipamentos de criticidade 1 das usinas. Apesar da manutenção dispor de planos de inspeção e preventivas, a natureza das falhas de instrumentos nem sempre pode ser detectada a olho nu. Os modos de falha muitas vezes representam uma degradação do sinal, congelamento de sinal, e/ou funcionamento intermitente. O desafio é conseguir monitorar estas falhas com o mínimo desenvolvimento de sistemas possível e o com o menor custo de implantação. Seguindo esta linha de raciocínio, oportunidades de uso de sistemas existentes foram vislumbradas nas ferramentas já implementadas para controle de malhas. Os profissionais de operação, processo e automação da usina de Alegria formaram um grupo de trabalho visando minimizar transbordo de tanques, onde ferramentas de software (PlantTriage) já vinham sendo utilizadas para monitorar o tempo de operação das malhas em automático, o erro e a saturação das malhas de controle. É importante ressaltar que alguns sistemas de automação possuem recursos de diagnóstico para manutenção, para variáveis discretas, entretanto, para variáveis analógicas, não há no mercado este tipo de recurso. Sabemos que os instrumentos que trabalham em rede possuem diagnósticos que indicam a saúde desses ativos, mas nos equipamentos que trabalham com sinais de 4 a $20 \mathrm{~mA}$ não possuímos nenhuma informação além do sinal de medição de campo.

\section{MATERIAIS E MÉTODOS}

\subsection{PIMS}

O PIMS (Plan Information Management System) apresenta-se como uma solução direta para o problema de falta de acessibilidade às informações e ao conhecimento efetivo do processo industrial. Este sistema é caracterizado pela capacidade de coletar e centralizar dados de diferentes unidades da planta em uma base única, armazenando-os por vários anos e disponibilizando-os a diferentes níveis de usuários sob forma de aplicações de alto valor para monitoramento e análise do processo de produção. 
Podem ser apresentadas como principais características dos Sistemas PIMS:

- Coleta de dados de sistemas de chão-de-fábrica e sistemas corporativos;

- $\quad$ Alta e eficiente compressão dos dados;

- Capacidade de armazenamento histórico superior a 10 anos;

- Alta velocidade de resposta a consultas;

- Ferramentas clientes de utilização fácil e intuitiva, tais como telas sinóticas, relatórios, KPIs(Indicador-chave de desempenho), portal web, dentre outros.

\subsection{MS-SQL Server}

O MS-SQL Server é um SGBD - sistema gerenciador de banco de dados relacional desenvolvido pela Microsoft. Como um banco de dados, é um produto de software cuja função principal é a de armazenar e recuperar dados solicitados por outras aplicações seja no mesmo computador ou em execução em outro computador através de uma rede. O MS-SQL Server é utilizado para diferentes cargas de trabalho (variando de pequenas aplicações que armazenam e recuperam dados no mesmo computador, a milhões de computadores que manipulam grandes quantidades de dados a partir da Internet ao mesmo tempo).

\subsection{ICD de Saúde de ativos}

Idealizado na Usina de Alegria, Complexo de Mariana - MG, esse algoritmo de acompanhamento do instrumento baseia-se em algumas premissas de funcionamento e em função destas informações resulta em uma nota de desempenho e sinalização da Saúde e necessidade de intervenção.

Os instrumentos a serem acompanhados podem ser do tipo analógicos, convencionais $(4-20 \mathrm{~mA})$, que medem variáveis de processo contínuas (ex. massa, pressão, densidade, posição), ou analíticos (analisadores), que realizam a análise de uma amostra do processo para medir uma ou mais variáveis de processo contínuas (ex. granulometria, umidade, teor de ferro).

Partindo do conceito de OIE (Overall Instrument Effectiveness), derivado do OEE (Overall Equipment Effectiveness), foram feitas analogias entre as características de cada elemento (disponibilidade, utilização, desempenho) com as características do ativo de automação. A função de um instrumento de campo, independentemente do seu tipo e da tecnologia é medir bem, para isso:

Além da análise da variável de processo, é possível inferir alguns estados básicos dos instrumentos:

Congelamento da variável: Avaliar se a variável está com valor fixo, sem nenhuma variabilidade por um período de tempo determinado. O congelamento da leitura pode ser ocasionado por sensor do instrumento incrustados/emperrado, ou outra falha no instrumento, por exemplo.

Saturação: variável com valor fora da faixa (limites predefinidos) ou repetidamente fornecendo valores máximos ou mínimos por um período de tempo determinado.

Perda de Comunicação (Abertura de loop): variável com valor fixo ou com um valor não numérico (Bad) por um período de tempo determinado, ou detecção através do próprio cartão analógico. 


\subsection{Detalhamento dos Indicadores Chave de Desempenho [2]}

Vale lembrar que o ICD de instrumentação se baseia no conceito de OEE (Overall Equipment Effectiveness ou Índice Global de Eficiência dos Equipamentos), que por sua vez, é um indicador que expressa o percentual de utilização do equipamento na sua plenitude, considerando a situação ideal de velocidade máxima, sem paradas, sem desvios e sem reprocessos, com qualidade total.

Com base nisso, os principais conceitos a serem utilizados na definição dos indicadores são:

- DF: Disponibilidade física do ativo: pode ser calculado a partir da disponibilidade de dados armazenados nos sistemas historiadores (PIMS).

o Valores disponíveis: 0 a 1;

o Origem: Valor de qualidade do dado histórico;

- Valor $=0$ para valores não numéricos ou inexistentes;

- Valor $=1$ para valores numéricos

- U: Utilização do ativo: indicação de utilização através da leitura analógica

o Valores disponíveis: 0 a 1;

o Origem: Análise da variação da leitura (desvio padrão) do instrumento no tempo;

o Valor $=0$ para leitura congelada ou com desvio elevado

o Valor $=1$ para Leitura com variação normal;

- D: Desempenho do ativo: Indicação de desempenho através da leitura analógica:

o Valores disponíveis: 0 a 1;

- Origem: Comparação da leitura com parâmetros (zero e span)

- Valor = 0 para condição de saturação máxima (span) ou mínima (zero);

o Valor= 1 para ausência de condição de saturação máxima (span) ou mínima (zero);

\subsection{Metodologia de Cálculo dos Indicadores}

Para o cálculo dos indicadores, considera-se as seguintes definições:

- Intervalo de cálculo: os ICDs serão calculados a partir do histórico das variáveis num intervalo de tempo de 1 hora, ou seja, a cada 1 hora será gerado um novo valor para cada ICD referente a janela de 1 hora na base histórica de dados.

- Dados históricos dos ICDs: os valores gerados para os ICDs serão armazenados num banco de dados de forma a permitir seu monitoramento e agregação em diferentes bases de tempo (diária, semanal, mensal, etc).

- Condição de cálculo: para calcular um ICD, deve-se avaliar se o processo e/ou equipamento principal associado a cada instrumento está no regime normal de operação ou em funcionamento. Para isso, deve-se atribuir uma condição de processo em operação para atuar como uma condição de filtro de pesquisa dos dados históricos dos instrumentos.

- Tempo de processo em operação (Tonline): refere-se ao tempo total (\%) de funcionamento do processo e/ou equipamento principal associado a cada instrumento em um intervalo de cálculo. Ficou definido que o instrumento 
será avaliado (ou seja, será calculado o ICD) somente se o tempo de processo em operação for igual ou superior a $90 \%$ do intervalo de cálculo.

- Span: trata-se do valor máximo de medição de cada instrumento. Este parâmetro, extraído da base de configuração do PIMS, é usado no cálculo do ICD de saturação

- Zero: trata-se do valor mínimo de medição de cada instrumento. Este parâmetro, extraído da base de configuração do PIMS, é usado no cálculo do ICD de saturação

- Desvio padrão $(\delta)$ : determinado a cada intervalo de cálculo, trata-se do valor de desvio padrão da medição de cada instrumento enquanto a condição de cálculo estiver satisfeita.

- Saturação máxima (SMax): determinado a cada intervalo de cálculo, trata-se do percentual de tempo em que a medição do instrumento é igual ou superior ao Span.

- Saturação mínima (Smin): determinado a cada intervalo de cálculo, trata-se do percentual de tempo em que a medição do instrumento é igual ou inferior ao Zero.

- Perda de comunicação (PCom): determinado a cada intervalo de cálculo, trata-se do percentual de tempo de funcionamento do processo e/ou equipamento principal em momentos onde não há registro de dados históricos da variável de medição

- Congelamento (C): determinado a cada intervalo de cálculo, trata-se do percentual de tempo de funcionamento do processo e/ou equipamento principal em momentos onde o desvio padrão é inferior a uma tolerância, dada por: (Span - Zero) * $0,024 \%$, onde $0,024 \%$ é a resolução do instrumento (1/4096).

- Perturbação $(P)$ : determinado a cada intervalo de cálculo, trata-se do percentual de tempo de funcionamento do processo e/ou equipamento principal em momentos onde o desvio padrão é superior a um valor limite, dada por: (Span - Zero) ${ }^{*} 20 \%$, onde $20 \%$ é um valor empírico.

Com base nas definições acima, o cálculo dos ICDs devem seguir as seguintes equações:

- Disponibilidade física (Equação 1):

$$
D F=\frac{T_{\text {online }}-P_{\text {Com }}}{T_{\text {online }}}
$$

- Utilização (Equação 2):

$$
U=\frac{T_{\text {online }}-C-P}{T_{\text {online }}}
$$

- Desempenho (Equação 3):

$$
D=\frac{T_{\text {online }}-S_{\max }-S_{\min }}{T_{\text {online }}}
$$


Neste aplicativo, serão considerados dois tipos de indicadores agregados para determinar a saúde dos instrumentos:

- OIE (OEE para instrumentação) (Equação 4):

$$
O I E=0,333 * D F+0,333 * D F * U+0,333 * D F * U * D
$$

- Rendimento Operacional para instrumentação (RO) (Equação 5):

$$
\mathrm{RO}=\mathrm{DF} * \mathrm{U}(5)
$$

Portanto, a tabela abaixo resume os ICDs e seus respectivos níveis hierárquicos (Tabela 1).

Tabela 1. ICDs e níveis hierárquicos

\begin{tabular}{lll}
\hline \multicolumn{1}{c}{ Nível } & \multicolumn{1}{c}{ ICD } \\
\hline & $\bullet$ & Saturação máxima (Smax) \\
$1^{\circ}$ nível (básico) & $\bullet$ Saturação mínima (Smin) \\
& $\bullet$ Perturbação (P) \\
& $\bullet$ Perda de comunicação (Pcom) \\
\hline \multirow{2}{*}{$2^{\circ}$ nível (intermediário) } & $\bullet \mathrm{UF}$ \\
\hline $3^{\circ}$ nível (agregado) & $\bullet$ & $\mathrm{D}$ \\
\hline
\end{tabular}

\section{RESULTADOS E DISCUSSÃO}

Foi criado um aplicativo que calcula as estatísticas (média ponderada no tempo e desvio padrão), o tempo de perda de comunicação, o tempo de congelamento, o tempo com perturbação, o tempo em saturação máxima e o tempo em saturação mínima relativo aos dados colhidos e filtrados do PIMS e grava em uma tabela do banco de dados MS-SQL server. De posse destes dados armazenados no banco, são gerados diversos relatórios que auxiliam os inspetores na análise e diagnóstico dos instrumentos por período, área, processo ou tipo de instrumento (nível, vazão, etc.)

Abaixo, segue um exemplo de um ganho do sistema, auferido em uma das usinas.

\subsection{Pesquisa do Problema}

Diagnostico e apoio do \% de Fe no rejeito da Linha 2 da Flotação (Figura 1). 


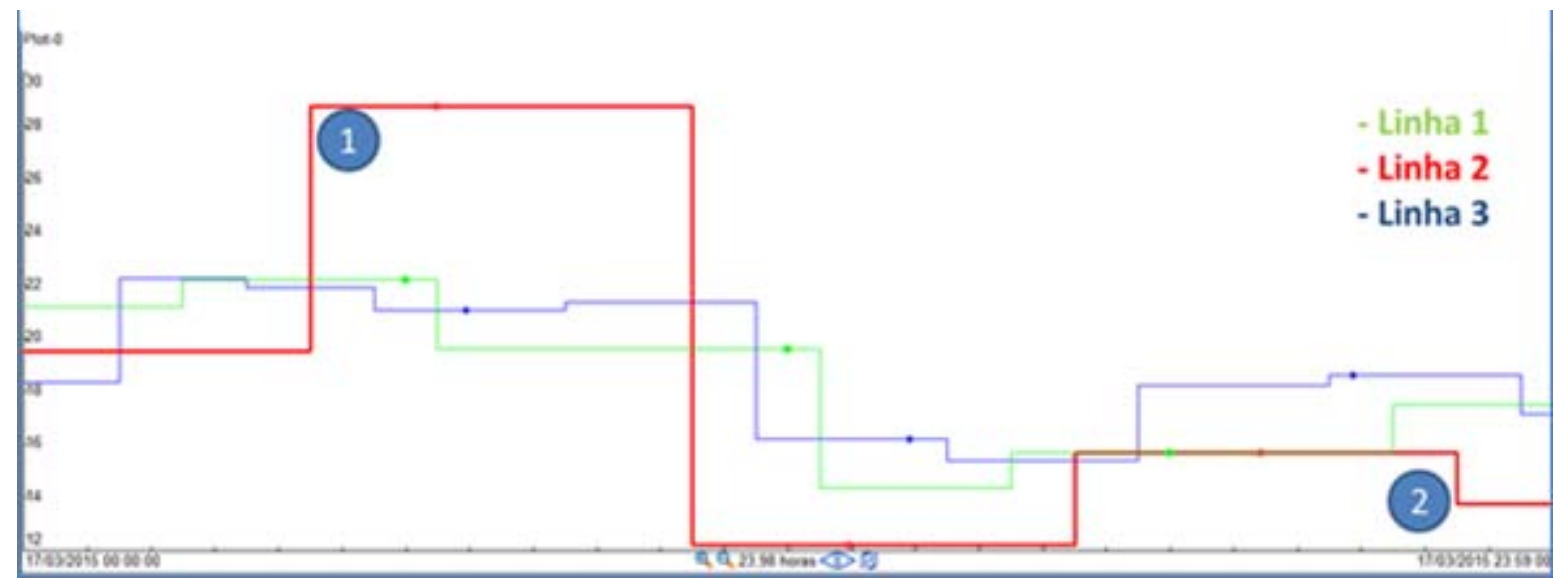

Figura 1. \% Fe no rejeito das linhas da flotação.

(No início do dia identificamos, em paralelo à operação, um alto teor de ferro no rejeito da Linha 2 da Flotação (em torno de 28\%). As demais linhas indicavam cerca de $22 \%$.

Fizemos contato com os operadores da usina e conversamos sobre alguns itens e aspectos impactantes. Dentre eles, destacamos: excesso de recirculação de massa (vazão maior que as demais) causando transbordo na BP1430CN014, algumas células que não respondiam ao pedido de set point e uma dosagem de amina zerada na linha 1 (defeito na válvula dosadora). Juntamente com demais diagnósticos que a operação já havia realizado e em contato presencial com o engenheiro de controle e automação, algumas ações foram tomadas.

\subsection{Solução Implantada}

\subsubsection{Manutenção condicional}

Controle - Redução ferro Rejeito. Correção BP1430CN014 - Válvula controle de amina. Março 2015

$22 \%$ Fe Rejeito $\rightarrow 17,6 \%$ Fe Rejeito

\subsubsection{Redução de perda convertida em PF - 360t - US\$ 5.400}

Revisão das malhas de controle de nível das células que não respondiam ao pedido de set point e malha de dosagem de amina com vazão obstruída na linha 1 por defeito na válvula dosadora.

\subsection{Ganho Estimado}

Diagnostico e apoio do \% de Fe no rejeito da Linha 2 da Flotação de Conceição II. (2) Ao final do dia o resultado médio do rejeito dessa linha fechou em $17,6 \% \mathrm{Fe}$. $\mathrm{O}$ mais baixo em comparação às demais linhas, sendo $17,86 \%$ na Linha 1; e 19,13\% na Linha 3.

\section{CONCLUSÃO}

Através do aplicativo criado para o monitoramento dos indicadores de inspeção preditiva dos ativos de instrumentação, podemos perceber antecipadamente, de maneira descentralizada e sem que o inspetor de manutenção tenha que sair a campo verificando instrumento por instrumento se existem medições que estão congeladas, com ruído (variações bruscas nas medições dos instrumentos em curtos 
períodos de tempo), trabalhando sob saturação de fundo de escala ou que tenham perdido a comunicação com o sistema de armazenamento de dados (PIMS). Esta metodologia ajuda a reduzir o tempo de paradas corretivas por motivo de problemas com as medições da instrumentação.

\section{Agradecimentos}

Houve uma participação ativa e importante das áreas de Operação, Manutenção e Automação, o que contribuiu positivamente para o sucesso na implantação e utilização do sistema desenvolvido.

\section{REFERÊNCIAS}

1 DIFS - Diretoria de Ferrosos Sudeste - Recomendações para Implementação de Indicadores de Desempenho - Função Instrumentação. Documento interno Vale.

2 Requisição Técnica - Detalhamento e Implantação - Função Instrumentação. Documento interno Vale. 